\title{
Propagating collective excitations in molten salts*
}

\author{
T.Bryk, I.Mryglod \\ Institute for Condensed Matter Physics \\ of the National Academy of Sciences of Ukraine, \\ 1 Svientsitskii Str., 79011 Lviv, Ukraine
}

Received May 6, 2003

Longitudinal as well as transverse dynamics of a molten salt beyond the hydrodynamic region is studied within the generalized collective modes (GCM) approach. An analytical three-variable model is applied to the treatment of the coupled long- and short-time charge fluctuations. Dispersion laws of propagating kinetic collective excitations such as optic phonon-like modes, heat and shear waves are obtained and analyzed for the case of molten $\mathrm{NaCl}$ within the eight-variable GCM scheme, combining the analytical methods and molecular dynamics simulations.

Key words: generalized hydrodynamics, collective excitations, ionic liquids, molten salts, longitudinal and transverse dynamics

PACS: $05.20 . J j, 61.20 . L C$

\section{Introduction}

So far, the picture of collective dynamics in molten salts has been well established exclusively in the hydrodynamic regime [1-3], i.e. reflecting the processes on large spatial and time scales. The hydrodynamic four-variable model permits to obtain analytic expressions for hydrodynamic time correlation functions and the spectrum of hydrodynamic collective excitations $[4,5]$. This model can explain a very specific feature of the relaxational behaviour of molten salts which appears due to the fluctuations of an inner electrical field under the constraint of total electric neutrality of the system and makes the main difference from the case of non-ionic binary mixtures. In particular, in reference [4] it was found that, depending on the value of electrical conductivity $\sigma$, two different regimes in the relaxational behaviour of a molten salt can be observed. Namely, for the systems with large values of conductivity, the charge fluctuations are practically uncoupled from the mass fluctuations, which makes the latter isomorphous to those of an one-component neutral fluid.

*Dedicated to Myroslav Holovko on occasion of his 60 th birthday. 
When $\sigma$ is small enough, the relaxational behaviour of a molten salt becomes isomorphous to that of a neutral mixture, with electrical conductivity playing a role similar to the mutual diffusion.

The hydrodynamic set of longitudinal dynamical variables for a molten salt consists of four microscopic operators of total mass-density $n_{t}(k, t)$, charge density $n_{q}(k, t)$, longitudinal total mass-current $J_{t}^{\mathrm{L}}(k, t)$ and total energy $e(k, t)$ :

$$
\mathbf{A}^{(\text {hyd })}(k, t)=\left\{n_{t}(k, t), n_{q}(k, t), J_{t}^{\mathrm{L}}(k, t), e(k, t)\right\} .
$$

These four operators correspond to the fluctuations of conserved quantities and describe the slowest dynamical processes in ionic melts. The mutual charge current fluctuations are associated with the fast processes of a shorter time scale, compared with the hydrodynamic ones, and are not taken into account within the standard hydrodynamic treatment.

During the recent few years a systematic approach to the studies of collective dynamics in pure and binary liquids, based on the concept of generalized collective excitations [6], was undertaken [7-11]. Within the generalized collective mode (GCM) approach, proposed originally in reference [6] and developed then in references [12-15], the collective excitations in the fluids are directly connected with eigenvalues of the so-called generalized hydrodynamic matrix, which determines the time evolution of the system. Such a definition of collective modes is in agreement with the generally accepted principle of statistical physics, when the collective modes are identified with the poles of the relevant Green function or generalized susceptibility.

One of the most important advantages of the GCM approach is a possibility of a detailed theoretical analysis of time correlation functions obtained in molecular dynamics (MD) simulations. The MD-derived functions contain in their very shape the information about all possible long- and short-time scale processes (diffusivelike, propagating and kinetic collective modes) as well as mode-coupling effects in a liquid. An analysis based on hydrodynamic set of equations fails to explain the shape of MD-derived functions in a wide range of wavenumbers $k$ and frequencies $\omega$. In contrast to the standard hydrodynamics, the GCM method, being one of the most consistent ways of constructing the generalized hydrodynamics, treats on the same footing the long- and short-time scale processes: the generalized Langevin equation for time correlation functions is solved in the matrix form using the basis set of dynamical variables, which apart from hydrodynamic (slow) variables contains their time derivatives (fast variables). The time evolution of these fast or so-called "extended" variables which describe the processes of the time scale shorter than the hydrodynamic ones, is obtained in computer experiments in order to estimate relevant time correlation functions and static averages. In general, the basis set of $N_{v}$ dynamical variables generates $N_{v} \times N_{v}$ secular equation and results in $N_{v}$ generalized collective modes (eigenvalues). Among the $N_{v}$ eigenvalues, the lowest ones in the long-wavelength region always correspond to the hydrodynamic modes, which have a right asymptotic behaviour in the small $k$ limit predicted by the linear hydrodynamics. All other eigenvalues are called kinetic modes and correspond to the 
processes of short-time scale. They cannot be obtained within the standard hydrodynamic treatment. Kinetic collective excitations sufficiently affect all the dynamical quantities beyond the hydrodynamic region. However, theoretical studies focused on the kinetic collective excitations in liquids are rather rare.

The additional dynamic variables which reflect the mutual motion of species make a noticeable difference in theoretical models for simple and binary fluids (for molten salts these are charge density $n_{q}(k, t)$ and charge current $\mathbf{J}_{q}(k, t)$ ). The dynamics of charge current (mass-concentration current in the case of non-ionic mixtures) fluctuations belong to kinetic-like processes. That is why the study of kinetic collective excitations caused by kinetic processes and their interaction with sound excitations within a generalized model would be of great interest. Recently, we studied within the GCM approach the transverse [9,10] and longitudinal [11] dynamics of Lennard-Jones and metallic mixtures and found that the optic-like excitations, which by dispersion law resemble the charge waves in molten salts, can exist even in non-ionic binary liquids. It was shown that the damping of mass-concentration propagating modes is very important in estimating their dispersion relations. In longwavelength limit the damping of optic-like excitations is determined by the value of mutual diffusion and the mass-concentration static structure factor $S_{x x}(k)$, which at $k=0$ is a measure of how close the binary non-ionic mixture is to the demixing. As a consequence, it was obtained that in the dense gas mixtures $\mathrm{He}_{75} \mathrm{Ar}_{25}$ and $\mathrm{He}_{65} \mathrm{Ne}_{35}$ with high mutual diffusion and large value of $S_{x x}(k=0)$ the long-wavelength opticlike excitations are suppressed and their branch does not exist in the spectrum of collective excitations for small wavenumbers.

In this paper we report the GCM study of longitudinal and transverse dynamics of a molten salt $\mathrm{NaCl}$ with the main attention focused on the dispersion relation of kinetic collective excitations, and optic-like (charge) waves, in particular. The remaining part of the paper is organized as follows. In section 2 we analytically solve the generalized Langevin equation in Markovian approximation using a threevariable model for charge fluctuations. In section 3 the four- and eight-variable GCM approach is applied to the numerical analysis of the transverse and longitudinal dynamics of molten $\mathrm{NaCl}$, respectively, and section 4 contains conclusions of this study.

\section{Coupled long- and short-time charge fluctuations: an analytical treatment}

Let us start from the simplest models of collective charge dynamics in ionic liquids. This can be done based on the separate treatment of longitudinal dynamics of charge fluctuations using generalized Langevin equation in Markovian approximation. In our case, the separated treatment means that we neglect the coupling between the charge fluctuations and slow dynamic processes of another origin, such as thermal and mass-density fluctuations. Both kinds of fluctuations could cause additional mechanisms of damping for the charge fluctuations. Here, in order to investigate the physical sense of the "bare" charge collective modes, we focus exclu- 
sively on the coupling between slow and fast charge fluctuations. Such a simplified approach permits to obtain a very precious information on the lowest kinetic modes caused by charge fluctuations, and to analyze in detail the effects of interaction between them.

Let us consider the generalized Langevin equations for the following basis set of three dynamical variables

$$
\mathbf{A}^{(3 q)}=\left\{n_{q}(k, t), J_{q}^{\mathrm{L}}(k, t), \dot{J}_{q}^{\mathrm{L}}(k, t)\right\},
$$

which are responsible for the longitudinal long- and short-time scale charge fluctuations. The hydrodynamic variable in this basis set is only $n_{q}(k, t)$, while the other two dynamical variables, $J_{q}^{\mathrm{L}}(k, t)$ and $\dot{J}_{q}^{\mathrm{L}}(k, t)$, describe faster charge fluctuations.

As a starting point for the subsequent analysis one may choose the behaviour of "bare" relaxing charge mode $d^{0}(k)$ and optic-like propagating excitations (or charge waves) $z_{ \pm}^{0}(k)$, which can be easily found from the eigenvalues problem [8-11] for the separate sets of dynamic variables, i.e. when the coupling between them is not taken into account. Namely, within the GCM approach the eigenvalues $d^{0}(k)$ and $z_{ \pm}^{0}(k)$ can be obtained from the secular equations for the corresponding generalized hydrodynamic matrix $\mathbf{T}(k)$, calculated for two separated subsets of dynamical variables, respectively:

(i) $\mathbf{A}^{(1 q)}=\left\{n_{q}(k, t)\right\}$, which includes only the hydrodynamic variable $n_{q}(k, t)$ and corresponds to the slowest component of charge fluctuations; and

(ii) $\mathbf{A}^{(2 q)}=\left\{J_{q}^{\mathrm{L}}(k, t), \dot{J}_{q}^{\mathrm{L}}(k, t)\right\}$, describing faster dynamical processes, caused by charge fluctuations.

The "bare" relaxing eigenvalue (or mode) $d^{0}(k)$, derived for the single-variable subset $\mathbf{A}^{(1 q)}$, is given by the expression

$$
d^{0}(k)=\frac{1}{\tau_{q q}(k)},
$$

where $\tau_{q q}(k)$ is the generalized correlation time, associated with the solely chargecharge time correlation function:

$$
\tau_{q q}(k)=\frac{1}{F_{q q}(k, t=0)} \int_{0}^{\infty} F_{q q}(k, t) \mathrm{d} t
$$

There exists an analytical result for asymptotic behaviour (when $k \rightarrow 0$ ) of the hydrodynamic correlation time $\tau_{q q}(k)[4,3]$. In contrast to the results known for the correlation time, describing the concentration fluctuations in non-ionic liquids or liquid metallic alloys [16], the correlation time $\tau_{q q}(k)$ at $k \rightarrow 0$ tends to a nonzero positive value, namely,

$$
\lim _{k \rightarrow 0} \tau_{q q}^{\text {hyd }}(k) \simeq \frac{\varepsilon}{4 \pi}\left(\frac{1}{\sigma}+\frac{\lambda_{p}^{2} T}{\rho C_{p}}\right) \equiv \tau_{q q}(0)+\tau_{h q}(0),
$$

defined by conductivity $\sigma$ and thermoelectric effects (first and second term in (5), respectively; see reference [3] for details). 
The "bare" optic-like eigenvalues, derived for the two-variable subset $\mathbf{A}^{(2)}$, are purely imaginary ones

$$
z_{ \pm}^{0}(k)= \pm \mathrm{i}\left\langle\bar{\omega}_{k}^{4}\right\rangle^{\frac{1}{2}} \equiv \pm \mathrm{i} \omega(k),
$$

where the following shortcut

$$
\left\langle\bar{\omega}_{k}^{2 n}\right\rangle=\frac{\left\langle\omega_{k}^{2 n}\right\rangle}{\left\langle\omega_{k}^{2 n-2}\right\rangle}
$$

was introduced for normalization of frequency moments of the charge-charge dynamical structure factor $S_{q q}(k, \omega)([17])$ :

$$
\left\langle\omega_{k}^{2 n}\right\rangle=\int_{-\infty}^{\infty} \omega^{2 n} S_{q q}(k, \omega) \mathrm{d} \omega .
$$

Purely imaginary eigenvalues describe the propagating collective excitations without damping. In real ionic systems, due to interaction with different kinds of dynamic processes, these modes have a nonzero damping coefficient or, in other words, the lifetime for these excitations is finite.

Our next task is to take into account the coupling between slow charge density fluctuations and short-time charge current fluctuations, and to find out how this coupling can affect the frequency and damping of optic-like excitations in the hydrodynamic limit. In the analytical approach we can restrict ourselves just to the consideration of the charge fluctuations within the set of dynamic variables (2). In fact, the dispersion and damping of optic-like excitations in molten salts is the result of interaction with total density and heat fluctuations as well. However, it is extremely difficult to perform an analytical analysis by taking into account the coupling between all possible "bare" excitations. All the microscopic processes between the generalized hydrodynamic and kinetic collective excitations are taken into account in our numeric approach performed within many-variable scheme in the next section. Simplified models of separated dynamics considered here permits to better understand the general tendencies of spectra formation in molten salts.

The generalized hydrodynamic matrix [15] constructed using the basis set of dynamical variables $\mathbf{A}^{(3 q)}$ for the description of solely charge fluctuations, can be written down in the following form:

$$
\mathbf{T}(k)=\left(\begin{array}{ccc}
0 & -\mathrm{i} k & 0 \\
0 & 0 & -1 \\
-\mathrm{i} k^{-1}\left(\left\langle\bar{\omega}_{k}^{4}\right\rangle-\left\langle\bar{\omega}_{k}^{2}\right\rangle\right) \tau_{q q}^{-1} & \left\langle\bar{\omega}_{k}^{4}\right\rangle & \left(\left\langle\bar{\omega}_{k}^{4}\right\rangle\left\langle\bar{\omega}_{k}^{2}\right\rangle^{-1}-1\right) \tau_{q q}^{-1}
\end{array}\right) .
$$

Three eigenvalues of the generalized hydrodynamic matrix $\mathbf{T}(k)$ are rather complicated for a detailed analysis. Therefore, we present them in a little bit simpler manner. Let us denote by $\delta$ the additional damping of the relaxing mode $d(k)$ caused by the coupling with optic-like excitations, so that:

$$
d(k \rightarrow 0) \equiv d^{0}+\delta=\frac{1}{\tau_{q q}^{0}}+\delta
$$

This means that instead of thermal correction $\tau_{h q}(k)$ for the correlation time (5), which appeared due to the coupling between charge and heat fluctuations within 
the hydrodynamic model, the three-variable model (2) produces another correction to $d(k)$ caused by the coupling between slow and fast charge fluctuations. Hence, immediately the eigenvalue problem for the generalized hydrodynamic matrix $\mathbf{T}(k)$ can be reduced [with a known purely real eigenvalue (8)] to the quadratic equation, and one gets for other two eigenvalues the following solutions:

$$
\left.z_{ \pm}(k)\right|_{k \rightarrow 0} \simeq \Gamma_{q} \pm \sqrt{\Gamma_{q}^{2}+\frac{\left\langle\bar{\omega}_{k}^{2}\right\rangle-\left\langle\bar{\omega}_{k}^{4}\right\rangle}{1+\tau_{q q}^{0} \delta}} .
$$

If the expression under the square root is negative, the eigenvalues $z_{ \pm}(k)$ describe a pair of complex-conjugated modes which correspond to the charge waves, propagating in opposite directions. Thus, due to the coupling with slow relaxing processes, connected with the electric conductivity, the frequency of optic-like excitations is somewhat modified in comparison with the "bare" solution (6), and one has:

$$
\omega(k \rightarrow 0)=\sqrt{\frac{\left\langle\bar{\omega}_{k}^{4}\right\rangle-\left\langle\bar{\omega}_{k}^{2}\right\rangle}{1+\delta \tau_{q q}^{0}}-\Gamma_{q}^{2}},
$$

where $\Gamma_{q}$ is the damping coefficient for the optic-like excitations:

$$
\Gamma_{q}=\frac{\left\langle\bar{\omega}_{k}^{4}\right\rangle-\left(2+\delta \tau_{q q}^{0}\right)\left\langle\bar{\omega}_{k}^{2}\right\rangle}{2 \tau_{q q}^{0}\left\langle\bar{\omega}_{k}^{2}\right\rangle} .
$$

One can see in (11) that in a molten salt with a high conductivity, the value of correlation time $\tau_{q q}^{0}$ at $k=0$ is very small, which causes a high damping of optic-like excitations (or charge waves). It is quite possible that optic-like excitations can be even suppressed in some molten salts with high conductivity $\sigma$. For large values of the damping coefficients $\Gamma_{q}$ the expression under the square root in equation (10) can become positive and this means that instead of a pair of complex-conjugated solutions one obtains two purely real eigenvalues describing kinetic relaxing modes. Similar results were previously found for nonionic binary mixtures (see, e.g., [11]). Note also that the expressions (8)-(11) can be easily connected with the results derived for mass-concentration collective excitations in nonionic binary mixtures [11] by setting: $\delta \rightarrow 0,\left\langle\bar{\omega}_{k}^{2}\right\rangle \sim k^{2}$, and $\tau_{q q}^{0} \sim k^{-2}$.

Thus, based on the simplified analytical analysis presented above, we can conclude that the damping of long-wavelength optic-like excitations is defined mainly by the electric conductivity $\sigma$ and the second and fourth order frequency moments. Depending on these quantities the dispersion law $\omega(k)$ of optic-like excitations in long-wavelength region can be essentially changed, in particular such modes can be suppressed in some molten salts with high conductivity $\sigma$.

\section{Longitudinal and transverse spectra of molten $\mathrm{NaCl}$}

\subsection{Calculation details}

Molecular dynamics simulations were performed in the standard microcanonical ensemble on a system of 1000 particles in a cubic box in order to obtain the time 
evolution of hydrodynamic and short-time extended dynamical variables:

$$
\mathbf{A}(k, t)=\left\{n_{t}(k, t), n_{q}(k, t), \mathbf{J}_{t}(k, t), \mathbf{J}_{q}(k, t), \varepsilon(k, t), \dot{\mathbf{J}}_{t}(k, t), \dot{\mathbf{J}}_{q}(k, t), \dot{\varepsilon}(k, t)\right\},
$$

where the total number density $n_{t}(k, t)$, charge density $n_{q}(k, t)$, total mass-current density $\mathbf{J}_{t}(k, t)$ and energy density $\varepsilon(k, t)$ belong to the most slow hydrodynamic variables. Time correlation functions and static averages were used to estimate the matrix elements of the generalized hydrodynamic matrix $\mathbf{T}(k)$, constructed on the variables $\mathbf{A}=\left\{A_{i}(k, t)\right\}$. Eight longitudinal and four transverse eigenvalues of the matrix $\mathbf{T}(k)$ were obtained for each wavenumber $k$ sampled in MD. They form the spectra $\left\{z_{j}(k)\right\}$ of generalized collective excitations in the GCM approach.

We have used the Tosi-Fumi potentials for simulations of $\mathrm{NaCl}$ at $1262 \mathrm{~K}$ [18]. The time step in MD simulations was $2 \cdot 10^{-15} \mathrm{~s}$. The long-range Coulomb interaction was taken into account via Ewald summation. Eleven wavenumbers with the smallest value of $k_{\min }=0.197 \AA^{-1}$ were sampled in computer simulations. Additional averaging over all possible directions of vectors $k$ were taken. The time evolution of basis dynamical variables was recorded over the production run of $3 \cdot 10^{5}$ time steps.

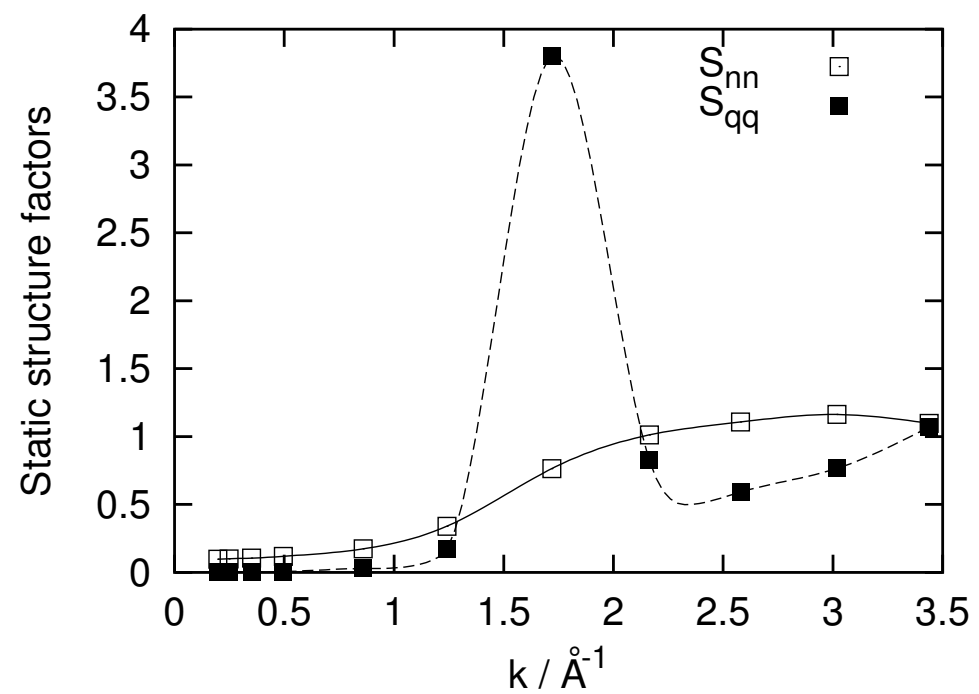

Figure 1. Density-density $S_{n n}(k)$ and charge-charge $S_{q q}(k)$ static structure factors directly evaluated in molecular dynamics.

In figure 1 the static structure factors "total density-total density"

$$
S_{n n}(k)=\left\langle n_{t}(k, 0) n_{t}^{*}(k, 0)\right\rangle
$$

and "charge density-charge density"

$$
S_{q q}(k)=4\left\langle n_{q}(k, 0) n_{q}^{*}(k, 0)\right\rangle
$$

are shown at the wavenumbers sampled in MD simulations. The factor 4 in the $S_{q q}(k)$ structure factor was used to normalize its short-wavelength asymptotics to unity. It 
is important to note the structureless feature of the $S_{n n}(k)$ structure factor, while charge fluctuations have a well-pronounced first peak of the relevant structure factor located at $k=1.7 \AA^{-1}$. This makes the main difference in the behaviour of static structure factors in molten $\mathrm{NaCl}$ in comparison, for instance, with the behaviour of $S_{n n}(k)$ and "mass-concentration - mass-concentration" structure factor $S_{x x}(k)$ in a Lennard-Jones equimolar KrAr mixture [9-11]. In the later case, the function $S_{x x}(k)$ exhibited almost a structureless shape and $S_{t t}(k)$ had a sharp first maximum. The effect of such a different behaviour of static structure factors on the spectra of longitudinal excitations will be discussed in the next paragraphs.

\subsection{Transverse dynamics}

Transverse dynamics in liquids is much simpler for the analysis than the longitudinal one because, in particular, the transverse components of dynamical variables do not interact with the heat fluctuations. In the case of transverse dynamics the basis set within the model (12) consists of four dynamical variables:

$$
\mathbf{A}^{(4 T)}=\left\{J_{t}^{T}(k, t), J_{q}^{T}(k, t), \dot{J}_{t}^{T}(k, t), \dot{J}_{q}^{T}(k, t)\right\} .
$$

Hydrodynamic treatment of the transverse dynamics is based only on a single variable $J_{t}^{T}(k, t)$ which is the transverse component of total mass-current. The imaginary parts of four eigenvalues, which was obtained for the generalized hydrodynamic matrix $\mathbf{T}(k)$, constructed on the basis set (13), is shown in figure 2 .

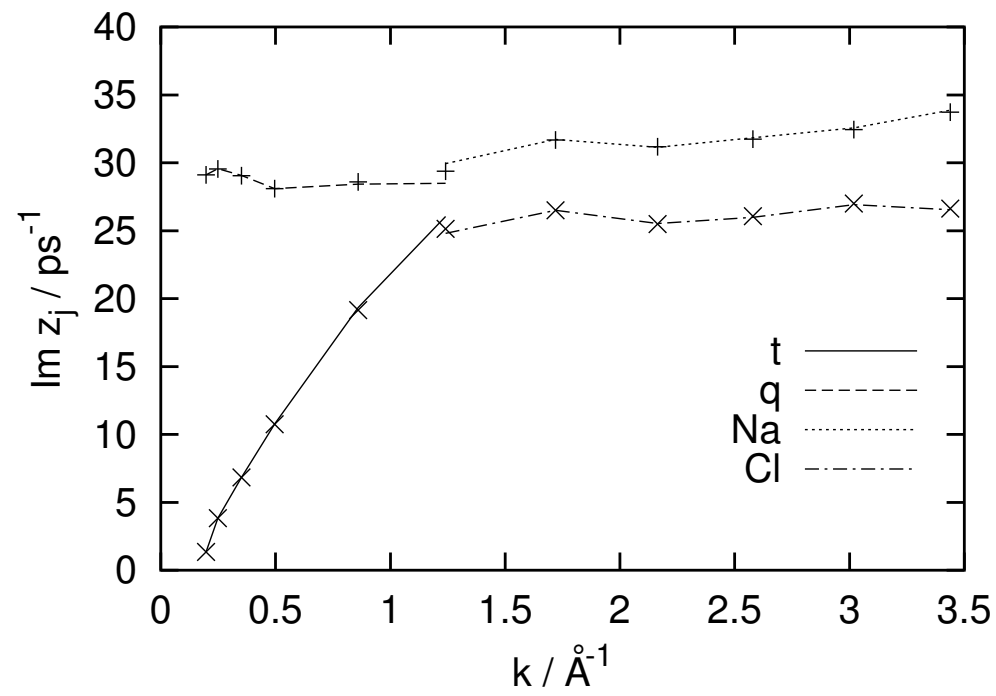

Figure 2. Dispersion of shear waves (crosses) and transverse optic-like excitations (symbols "+") obtained numerically in the four-variable $\left(\mathbf{A}^{(4 T)}\right)$ GCM approach for $\mathrm{NaCl}$. Lines correspond to the eigenvalues obtained by separated treatment of the "t-q" and "partial" dynamics with the help of the following sets of transverse variables: $\mathbf{A}^{(2, t)}$ (solid line), $\mathbf{A}^{(2, q)}$ (dashed line), $\mathbf{A}^{(2, \mathrm{Na})}$ (dotted line), $\mathbf{A}^{(2, \mathrm{Cl})}$ (dash-dotted line). 
For all the wavenumbers sampled in this study, we obtained two complex-conjugated pairs of eigenvalues which correspond to the propagating collective excitations. Since the hydrodynamic picture of transverse dynamics is reflected by a single relaxing mode, one can note that the smallest wavenumber reached in this study is located, in fact, outside the hydrodynamic region which is very small. However, the $k_{\min }$ is close enough to the hydrodynamic region because the dispersion of the lowfrequency branch in figure 2 implies the boundary of a propagation gap for shear waves for $k<k_{\min }$. To find the origin of each branch we have used the same treatment of transverse dynamics as we did before for the case of metallic alloys and Lennard-Jones mixtures (see, for details, [9]). Namely, the basis set $\mathbf{A}^{(4 T)}$ was divided into two subsets in two different ways: (i) we separated the dynamical variables reflecting mass-current and charge-current fluctuations

$$
\begin{aligned}
& \mathbf{A}^{(2, t)}=\left\{J_{t}^{T}(k, t), \dot{J}_{t}^{T}(k, t)\right\}, \\
& \mathbf{A}^{(2, q)}=\left\{J_{q}^{T}(k, t), \dot{J}_{q}^{T}(k, t)\right\},
\end{aligned}
$$

and, (ii) we split up the set $\mathbf{A}^{(4 T)}$ on the partial dynamical variables as follows:

$$
\begin{aligned}
\mathbf{A}^{(2, \mathrm{Na})} & =\left\{J_{\mathrm{Na}}^{T}(k, t), \dot{J}_{\mathrm{Na}}^{T}(k, t)\right\}, \\
\mathbf{A}^{(2, \mathrm{Cl})} & =\left\{J_{\mathrm{Cl}}^{T}(k, t), \dot{J}_{\mathrm{Cl}}^{T}(k, t)\right\} .
\end{aligned}
$$

These cases of two-variable subsets (14)-(15) were considered separately and the obtained numerical results are shown by lines in figure 2 (explanation is given in the capture). It is seen in figure 2 that in some restricted regions of wavenumbers a very good correspondence between two-variable and four-variable treatment (13) is observed. Namely, one can immediately conclude that there exist two different domains in the $k$-space, where such a correlation is very good. In long-wavelength region the transverse dynamics of a molten salt can be nicely reproduced in terms of weakly interacted shear waves (low-frequency branch, solid line) and optic-like excitations (high-frequency branch with almost flat dispersion, dashed line). In this region one can claim about collective dynamic behaviour. For larger $k, k>1.2 \AA^{-1}$, the transverse dynamics can be represented as a superposition of the partial transverse waves defined by the motion of light $(\mathrm{Na})$ and heavy $(\mathrm{Cl})$ components [dotted and dashed-dotted lines in figure 2, respectively]. This is the region of the so-called partial dynamics. It is interesting that such a picture for the transverse dynamics in molten salts corresponds quite well to acoustic and optic phonons in ionic crystal where on the first Brillouin zone boundary the relevant branches are mainly defined by heavy and light components, respectively.

\subsection{Longitudinal dynamics}

The spectrum of longitudinal collective excitations was obtained by solving the eigenvalues problem for the $8 \times 8$ generalized hydrodynamic matrix $\mathbf{T}(k)$, constructed on the following basis set of dynamic variables:

$$
\mathbf{A}^{(8 L)}=\left\{n_{t}(k, t), n_{q}(k, t), J_{t}^{\mathrm{L}}(k, t), J_{q}^{\mathrm{L}}(k, t), \varepsilon(k, t), \dot{J}_{t}^{\mathrm{L}}(k, t), \dot{J}_{q}^{\mathrm{L}}(k, t), \dot{\varepsilon}(k, t)\right\} .
$$


The results of our calculations, obtained for the dispersion of propagating longitudinal modes, are shown in figure 3. In contrast to the case of transverse dynamics we have obtained three branches of propagating collective excitations for $k>0.3 \AA^{-1}$. The lowest-frequency branch (shown by boxes) corresponds to heat waves, because it can be quite well reproduced by considering a separated treatment of the heat fluctuations on the basis subset $\{h(k, t), \dot{h}(k, t)\}$, where $h(k, t)$ is a dynamical variable of heat density. The branch of heat waves has a propagation gap in long-wavelength range in complete agreement with the standard hydrodynamics, because the heat waves belong to a class of kinetic collective excitations and cannot be obtained within the linear hydrodynamic approach. Another branch of collective excitations shown by crosses abides by almost linear dispersion law in long-wavelength region and describes the sound modes. One can see in figure 3 a small deviation from the linear dispersion law $c_{\mathrm{s}} k$ (dotted line) with $c_{\mathrm{s}}=3400 \mathrm{~m} / \mathrm{s}$, which can be attributed to the so-called positive dispersion due to the mode-coupling effects. This branch reaches at $k \approx 1.2 \AA^{-1}$ a region with a rather flat dispersion.

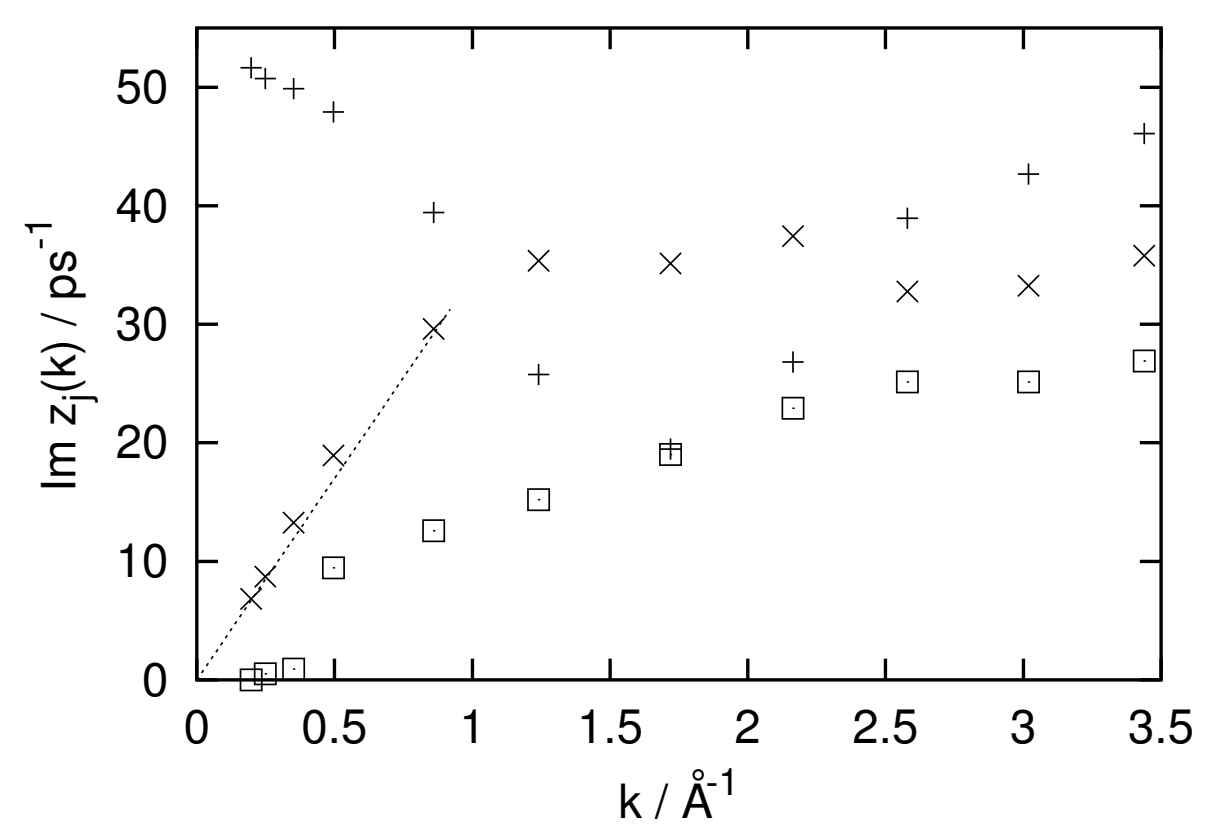

Figure 3. Dispersion of longitudinal collective excitations for molten $\mathrm{NaCl}$, obtained for the basis set $\mathbf{A}^{(8 L)}(k, t)$ [see (16)]: generalized sound modes (crosses), longitudinal optic-like excitations (symbols "+"), and heat waves (boxes), respectively. Dotted line shows the linear hydrodynamic dispersion law with the speed of sound $c_{\mathrm{s}}=3400 \mathrm{~m} / \mathrm{s}$.

The third branch of propagating excitations, shown in figure 3 by symbols "plus", tends to a value of $\sim 53 \mathrm{ps}^{-1}$ at $k \rightarrow 0$, and in the region of $k \approx 1.7 \AA^{-1}$ it has a wide minimum. In a similar manner as was described above, using the separated treatment of the total density and charge density fluctuations, it has been shown that the longitudinal propagating branches, shown in figure 3 by symbols "cross" 
and "plus", correspond to the acoustic and optic-like excitations and could be well reproduced on these separated subsets up to $k \approx 2 \AA^{-1}$. The wide minimum in the dispersion of optic-like excitations is closely related to a position of the first maximum in the static structure factor $S_{q q}(k)$. It is worth noting that in nonionic binary mixtures, for instance in the case of $\mathrm{KrAr}$ mixture [11], the wide minimum in the dispersion law is attributed to the acoustic branch and is connected mainly with the $k$-dependence of static structure factor $S_{n n}(k)$ (e.g., for KrAr this function has a sharp first maximum).

It is also interesting to compare the dispersion curves for transverse and longitudinal optic-like excitations in molten $\mathrm{NaCl}$, shown in figures 2 and 3 by symbols "plus". In contrast with nonionic binary mixtures, the main conclusion of such a comparison is the existence of a gap between $\mathrm{L}$ and $\mathrm{T}$ branches in long-wavelength limit, which is approximately equal to $25 \mathrm{ps}^{-1}$ for the case of $\mathrm{NaCl}$ studied. Such a gap in molten salts is a consequence of long-range Coulomb interaction between ions and has a complete analogy in ionic $\mathrm{NaCl}$ crystal.

\section{Conclusions}

Using the generalized collective modes approach, we have investigated the specific features of collective dynamics in a molten salt $\mathrm{NaCl}$ beyond the hydrodynamic region. We focused on the study of the propagating kinetic collective excitations which cannot be found within the standard hydrodynamic theory but play a crucial role in the dynamics of the system. In this way, three types of propagating collective modes were studied more in detail. Namely, optic-like excitations, heat and shear waves. The main results of this study can be summarized as follows:

(i) An analytical condition for the existence of long-wavelength optic-like excitations is derived within the three-variable analytical model describing the charge fluctuations. According to this condition, a high conductivity in molten salts can suppress the charge waves;

(ii) The spectrum of longitudinal collective excitations in $\mathrm{NaCl}$ was calculated numerically based on the eight-variable scheme of the generalized collective modes approach. It is shown, that this spectrum contains three branches of generalized propagating modes beyond the hydrodynamic region. Namely, we found one acoustic and two branches of kinetic propagating excitations describing the low-frequency heat waves and optic-like excitations;

(iii) Using the four-variable set for the case of transverse dynamics in $\mathrm{NaCl}$, we studied the spectrum of the generalized transverse excitations and found two branches of propagating collective modes: shear waves and optic-like excitations. Comparing the results obtained with those found for the separated subsets of dynamic variables, there is established the existence of two domains in $k$-space, in which the branches are of different character: short-wavelength domain of "partial" dynamics, and long-wavelength domain, where the collective type of dynamics is dominant;

(iv) In complete agreement with the results known for ionic crystals, we have 
found a gap between longitudinal and transverse optic-like excitations in molten $\mathrm{NaCl}$ in long-wavelength limit, caused by the long-range Coulomb interaction between ions. The obtained results are discussed in comparison with those known for nonionic binary mixtures.

\section{Acknowledgements}

I.M. thanks the Fonds zur Förderung der wissenschaftlichen Forschung (Austria) for financial support under Project No. P15247.

\section{References}

1. Parrinello M., Tosi M.P. // Rev. Nuovo Cim., 1979, vol. 2, No. 6.

2. March N.H., Parrinello M. Collective Effects in Solids and Liquids. Bristol, Hilger, 1982.

3. March N.H., Tosi M.P. Coulomb Liquids. London, NewYork, Academic Press, 1984.

4. Giaquinta P.V., Parrinello M., Tosi M.P. // Phys. Chem. Liq., 1976, vol. 5, p. 305.

5. Rovere M., Tosi M.P. // Rep. Prog. Phys., 1986, vol. 49, p. 1001.

6. de Schepper I.M., Cohen E.G.D., Bruin C., van Rijs J.C., Montfrooij W., de Graaf L.A. // Phys. Rev. A, 1988, vol. 38, p. 271.

7. Bryk T., Mryglod I., Kahl G. // Phys. Rev. E, 1997, vol. 56, p. 2903.

8. Bryk T., Mryglod I. // Phys. Lett. A, 1999, vol. 261, p. 349.

9. Bryk T., Mryglod I. // J. Phys.: Cond. Matt., 2000, vol. 12, p. 6063.

10. Bryk T., Mryglod I. // Phys. Rev. E, 2000, vol. 62, p. 2188.

11. Bryk T., Mryglod I. // J. Phys.: Cond. Matt., 2002, vol. 14, p. L445.

12. Omelyan I.P., Mryglod I.M. // Condens. Matter Phys., 1994, iss. 4, p. 128.

13. Mryglod I.M., Omelyan I.P., Tokarchuk M.V. // Mol. Phys., 1995, vol. 84, p. 235.

14. Mryglod I.M., Omelyan I.P. // Phys. Lett. A, 1995, vol. 205, p. 401.

15. Mryglod I.M. // Condens. Matter Phys., 1998, vol.1, p. 753.

16. March N.H., Tosi M.P. Atomic Dynamics in Liquids. Macmillan Press, 1976.

17. Hansen J.P., McDonald I.R. // Phys. Rev. A, 1975, vol. 11, p. 2111.

18. Ciccotti G., Jacucci G., McDonald I.R. // Phys. Rev. A, 1976, vol. 13, p. 426. 


\title{
Узагальнені колективні збудження у розплавах солей
}

\author{
Т.Брик, І.Мриглод \\ Інститут фізики конденсованих систем НАН України, \\ 79011 Львів, вул. Свєнціцького, 1
}

Отримано 6 травня 2003 р.

У методі узагальнених колективних мод (УКМ) досліджується повздовжня та поперечна динаміка розплавів солей поза областю гідродинамічної поведінки. В рамках простої тризмінної моделі аналітично проаналізовано особливості зв'язаної динаміки короткота довго-живучих зарядових флюктуацій. В рамках восьми змінного підходу методу УКМ, комбінуючи засоби аналітичної теорії та молекулярно-динамічні розрахунки, для розплаву $\mathrm{NaCl}$ отримано та проаналізовано дисперсійні закони для пропагаторних кінетичних колективних збуджень типу оптичних фононних мод, теплової та зсувної хвиль.

Ключові слова: узагальнена гідродинаміка, колективні збудження, іонні рідини, розплави солей, поперечна і повздовжня динаміка

PACS: 05.20.Jj, 61.20.LC 
